\title{
Energy Decay for a Degenerate Hyperbolic Equation with a Dissipative Term
}

\author{
By
}

\section{Fumihiko HirosawA*}

\section{§1. Introduction}

We shall investigate the energy decay of the solutions to the following Cauchy problem for a degenerate hyperbolic equation :

$$
\begin{gathered}
u_{t t}-a(t) \Delta u+2 b(t) u_{t}+m^{2} u=0, t \geq 0, x \in \mathbb{R}^{n} \\
u(0, x)=u_{0}(x), \mathrm{u}_{\mathrm{t}}(0, x)=u_{1}(x), x \in \mathbb{R}^{n}
\end{gathered}
$$

where $u(t, x)$ is real valued, $a(t) \in C^{1}([0, \infty)), a(t) \geq 0, b(t) \in C^{0}([0, \infty))$, $\inf _{t}\{b(t)\} \equiv b_{0}>0, \sup _{t}\{b(t)\} \equiv b_{1}<\infty, m$ is a positive constant and $\Delta$ is the Laplace operator in $\mathbb{R}^{n}$.

The purpose of this paper is to seek sufficient conditions on $a, b, m$ and the initial data $\left(u_{0}, u_{1}\right)$ which guarantee the exponential order decay of the total energy

$$
E(u(t))=\frac{1}{2}\left\{\left\|u_{t}(t)\right\|^{2}+a(t)\|\nabla u(t)\|^{2}+m^{2}\|u(t)\|^{2}\right\}
$$

to the solution of (1)-(2) as $t \rightarrow \infty$, where $\|\bullet\|$ denotes the usual $L^{2}\left(\mathbb{R}^{n}\right)$ norm.

Energy decay problem in the whole space for the wave equation with a dissipative term has been considered by many authors (Matsumura[2], Mochizuki [3], Mochizuki-Nakazawa [4], Rauch-Taylor[5], etc.). But it seems to be very few results for the case of general hyperbolic equations as (1).

If $a(t) \equiv 1$ and $b(t) \equiv 0$, the total energy $E(u(t))$ is conserved, that is, $E(u(t))$ $=E(u(0))$ for any $t$, and it is possible that the energy decays when $b \geq 0$. Indeed, it is well-known that the energy decays in exponential order if $\inf _{t}\{b(t)\}>0$ and $m$ $>0$ (see [5]), and decays like $O\left(t^{-1}\right)$ as $t \rightarrow \infty$ if there are positive constants $b_{0}$ and $b_{1}$ such that $b_{0}(1+t)^{-1} \leq b(t) \leq b_{1}, b^{\prime}(t) \leq 0$ and $m=0$ (see [3]; [2] and [4] have

Communicated by T. Kawai, April 23, 1998. Revised January 18, 1999.

1991 Mathematics Subject Classification(s) : 35L15, 35L80

* Institute of Mathematics, University of Tsukuba, Ibaraki 305-8571, Japan. 
gone into more particulars).

However, if $a(t) \not \equiv$ const., the energy does not always decay in spite of the existence of the dissipation, because $a(t)$ may play a part in the growth of the energy when $a^{\prime}(t)>0$. Indeed, according to Reissig-Yagdjian [6], there exists $a(t)$ $\in C^{1}([0, \infty))$ such that the total energy cannot be bounded for any function of $O\left(e^{\sigma t}\right)$ with $\sigma<1$. Thus, it seems that the behavior of the energy for the general hyperbolic equation is more complicate than that for the wave equation. Furthermore, we shall consider the case that $a(t)$ has zero points. When $a(t)$ vanishes at a point, in general, (1) is not $H^{\infty}$ well-posed in any neighborhood of the vanishing point. However, if the initial data is sufficiently regular, the Cauchy problem (1)-(2) is well-posed in the Gevrey class of order $3 / 2$ since $a(t) \in C^{1}$ (see $[1])$. Hence we can consider a classical solution of (1)-(2) and its energy for the initial data in the Gevrey class.

\section{§2. Preliminaries and Results}

In this section we mention our main theorems.

In the first theorem is treated the case that (1) is strictly hyperbolic, that is, $a(t)$ is strictly positive. In the second one, we consider the case that (1) is weakly hyperbolic, that is, $a(t)$ has a zero point.

We define the positive constants $\alpha_{0}=\alpha_{0}\left(b_{0}, b_{1}, m\right)$ and $\beta_{0}=\beta_{0}\left(b_{0}, b_{1}, m\right)$ by

$$
\alpha_{0}\left(b_{0}, b_{1}, m\right)= \begin{cases}b_{0} & \left(b_{0} b_{1}<m^{2}\right) \\ \frac{m^{2}}{b_{1}} & \left(b_{0} b_{1} \geq m^{2}\right)\end{cases}
$$

and

$$
\beta_{0}\left(b_{0}, b_{1}, m\right)= \begin{cases}b_{1}-\frac{m\left(b_{1}-b_{0}\right)}{\sqrt{m^{2}-b_{0}^{2}}} & \left(b_{0} b_{1}<m^{2}\right), \\ b_{1}-\sqrt{b_{1}^{2}-m^{2}} & \left(b_{0} b_{1} \geq m^{2}\right) .\end{cases}
$$

Theorem 2.1. Assume that $\inf _{t}\{a(t)\}>0, \sup _{t}\left\{\frac{a^{\prime}(t)}{2 a(t)}\right\}<\infty$, and $\left(u_{0}, u_{1}\right) \in H^{1} \times$ $L^{2}$. If the following condition

$$
\sup _{t}\left\{\frac{a^{\prime}(t)}{2 a(t)}\right\}<\alpha_{0}\left(b_{0}, b_{1}, m\right)
$$

holds, then there exists a positive constant $C_{\rho}$ such that the following decay estimate to the solution of the Cauchy problem (1)-(2) 


$$
E(u(t)) \leq C_{\rho} E(u(0)) e^{-2 \rho t}
$$

holds for any $t \geq 0$ and $\rho<\rho_{0}$, where

$$
\rho_{0}(I)=\beta_{0}-\left[\sup _{t \in I}\left\{\frac{a^{\prime}(t)}{2 a(t)}\right\}-\alpha_{0}+\beta_{0}\right]_{+}
$$

and $\rho_{0}=\rho_{0}([0, \infty))$.

Now, we shall introduce a class of functions, which is called the Gevrey class, to state our second theorem. Let $s>0$ and $\mu>0$. We define the Gevrey class $G_{\mu}^{s}$ and $G^{s}$ by

$$
\boldsymbol{G}_{\mu}^{s}=\left\{f(\boldsymbol{x}) \in \boldsymbol{H}^{\infty} ; \sup _{j}\left\{\frac{\mu^{j}}{j !^{s}}\left\|\nabla^{j} f\right\|\right\}<\infty\right\} \text { and } \boldsymbol{G}^{s}=\bigcup_{\mu>0} \boldsymbol{G}_{\mu}^{s}
$$

where

$$
\left\|\nabla^{j} f\right\|= \begin{cases}\left\|\Delta^{-\frac{J}{2}} f\right\| & (j: \text { even }), \\ \left\|\nabla \Delta^{\frac{j-1}{2}} f\right\| & (j: \text { odd })\end{cases}
$$

and $\Delta^{k}=\left(\partial_{x_{1}}^{2}+\cdots+\partial_{x_{n}}^{2}\right)^{k}$. Then we have the following theorem :

Theorem 2.2. Define the set $\mathfrak{I} \subset[0, \infty)$ by

$$
\mathfrak{I}=\left\{t \in[0, \infty) ; \frac{a^{\prime}(t)}{2 a(t)} \geq \alpha_{0} \text { or } a(t)=0\right\}
$$

Assume that there exist monotonically increasing sequences of non-negative real numbers $\left\{t_{j}\right\}$ and $\left\{t_{j}^{\prime}\right\}$ satisfying

$$
\mathfrak{I} \subset\left\{\begin{array}{ll}
\cup_{j \geq 1}\left(t_{j}, t_{j}^{\prime}\right) & \left(t_{1} \neq 0\right) \\
{\left[0, t_{1}\right) \cup \cup_{j \geq 2}\left(t_{j}, t_{j}^{\prime}\right)} & \left(t_{1}=0\right),
\end{array}\right\} \equiv \mathfrak{I}_{0},
$$

$$
\lim _{t \rightarrow \infty}\left\{\frac{\sum_{\left\{k ; t_{k} \leq t\right\}} a\left(t_{k}\right)^{-1}+\sum_{\left\{k ; t_{k}^{\prime} \leq t\right\}} a\left(t_{k}^{\prime}\right)^{-1}}{t}\right\}=0
$$

and

$$
\sum_{j}\left(t_{j}^{\prime}-t_{j}\right) \exp \left(M \sum_{l=1}^{j} \int_{t_{1}}^{t_{1}^{\prime}}\left[a^{\prime}(s)\right]_{+} d s\right)<\infty
$$

for any $M>0$. If $u_{0}, u_{1} \in G^{s}$ for $0<s<3 / 2$, then there exists a positive constant $C_{\rho, u_{0}, u_{1}}$ such that the following decay estimate to the solution of (1)-(2) 


$$
E(u(t)) \leq C_{\rho, u_{0}, u_{1}} e^{-2 \rho t}
$$

holds for any $\rho<\rho_{0}\left([0, \infty) \backslash \mathfrak{I}_{0}\right)$. Moreover, if

$$
\sum_{l \geq 1} \int_{t_{l}}^{t_{l}^{\prime}}\left[a^{\prime}(s)\right]+d s<\infty,
$$

then there exist positive constants $\mu$ and $C_{\rho}$ such that the following decay estimate of the infinity order energy holds

$$
\sum_{j=0}^{\infty} \frac{\mu^{j}}{j !^{\frac{3}{2}}} E\left(\nabla^{j} u(t)\right) \leq C_{\rho, u_{0}, u_{1}} e^{-2 \rho t}
$$

for any $\rho<\rho_{0}\left([0, \infty) \backslash \mathfrak{I}_{0}\right)$.

Furthermore, we can prove the following theorem by a little modification of the proof of Theorem 2.2.

Theorem 2.3. Under the same assumptions as in Theorem 2.2, there exists a positive constant $C_{l, \rho, u_{0}, u_{1}}$ such that the following decay estimate of the higher order energy

$$
E\left(\nabla^{l} u(t)\right) \leq C_{l, \rho, u_{0}, u_{1}} e^{-2 \rho t}
$$

holds for any $\rho<\rho_{0}\left([0, \infty) \backslash \mathfrak{I}_{0}\right)$ and for any positive integer $l$.

Remark 2.1. If the assumption (9) holds, then the measure of $\mathfrak{I}_{0}$ must be finite.

The proofs of our theorems are based on a well-known method to obtain the exponential order energy decay to the dissipative wave equation of Klein-Gordon type, which is introduced in Zuazua [7], for instance.

Let us consider the case that $a(t) \equiv 1$ and that $u(t, x)$ is real valued. Let $\alpha$ be a positive constant satisfying $m>\alpha$ and define $E_{(\alpha)}(u(t))$ by

$$
\begin{aligned}
& E_{(\alpha)}(u(t))=E(u(t))+\alpha\left(u(t), u_{t}(t)\right) \\
& =\frac{1}{2}\left\{\left\|u_{t}(t)+\alpha u(t)\right\|^{2}+\|\nabla u(t)\|^{2}+\left(m^{2}-\alpha^{2}\right)\|u(t)\|^{2}\right\},
\end{aligned}
$$

where $(\bullet, \cdot)$ denotes the usual $L^{2}\left(\mathbb{R}^{n}\right)$ inner product.

Differentiating $E_{(\alpha)}(u(t))$ with respect to $t$, using the equation (1) and applying Schwarz' inequality (1), we have

$$
\frac{d}{d t} E_{(\alpha)}(u(t))=-(b(t)-\alpha)\left\|u_{t}(t)\right\|^{2}-\alpha\|\nabla u(t)\|^{2}-\alpha m^{2}\|u(t)\|^{2}
$$




$$
\begin{aligned}
& -\alpha b(t)\left(u(t), u_{t}(t)\right) \\
\leq & -\left\{b(t)-\alpha\left(1+\frac{b(t)}{2 \alpha}\right)\right\}\left\|u_{t}(t)\right\|^{2} \\
& -\alpha\|\nabla u(t)\|^{2}-\alpha\left(m^{2}-\frac{\alpha b(t)}{2}\right)\|u(t)\|^{2},
\end{aligned}
$$

where $\alpha$ is a positive constant satisfying $\frac{2 m^{2}}{b(t)}>\alpha$. Now, we choose $\alpha$ satisfying

$$
\inf _{t}\left\{\frac{b(t)}{1+\frac{b(t)}{2 \delta}}\right\}>\alpha>0
$$

Then, there exists a positive constant $\rho$ such that

$$
\frac{d}{d t} E_{(\alpha)}(u(t)) \leq-\rho E_{(\alpha)}(u(t))
$$

hence we obtain

$$
E_{(\alpha)}(u(t)) \leq E_{(\alpha)}(u(0)) e^{-\rho t}
$$

Recalling the definition of $E_{(\alpha)}(u(t))$ and applying Schwarz' inequality, there exists a positive constant $C_{\alpha}$ such that we have from the above inequality

$$
E(u(t)) \leq C E(u(0)) e^{-\rho t}
$$

for any $t \in[0, \infty)$.

Thus, choosing $\alpha>0$ suitably, we obtain the energy decay of exponential order to the Cauchy problem (1)-(2) with $a(t) \equiv 1$.

Here we note the method above cannot be applied in case that $a(t)$ is not constant, but considering the choice of constant $\alpha$, it is possible to prove the exponential order decay to the solution of a general hyperbolic equation like (1).

\section{§3. Proof of Theorem 2.1}

Let $\alpha$ be a positive constant satisfying $m>\alpha$ and define $E_{(\alpha)}(u(t))$ by

$$
\begin{aligned}
E_{(\alpha)}(u(t)) & =E(u(t))+\alpha\left(u(t), u_{t}(t)\right) \\
& =\frac{1}{2}\left\{\left\|u_{t}(t)+\alpha u(t)\right\|^{2}+a(t)\|\nabla u(t)\|^{2}+\left(m^{2}-\alpha^{2}\right)\|u(t)\|^{2}\right\},
\end{aligned}
$$

where $(\bullet, \bullet)$ denotes the usual $L^{2}\left(\mathbb{R}^{n}\right)$ inner product. Here we remark that the energy norm $E_{(\alpha)}(u(t))$ is equivalent to the usual energy norm $E(u(t))$ since $\alpha<$ $m$, that is, there exists a positive constant $C_{\alpha}$ independent of $j$ such that the 
following inequality

$$
C_{\alpha}^{-1} E_{(\alpha)}\left(\nabla^{j} u(t)\right) \leq E\left(\nabla^{j} u(t)\right) \leq C_{\alpha} E_{(\alpha)}\left(\nabla^{j} u(t)\right)
$$

holds for any $j \geq 0$.

Let $\beta$ be a positive constant to be chosen later. Differentiating $E_{(\alpha)}(u(t))$ with respect to $t$, we have

$$
\begin{aligned}
\frac{d}{d t} E_{(\alpha)}(u(t))= & \frac{1}{2}\left(a_{t}(t)-2 \alpha a(t)\right)\|\nabla u(t)\|^{2} \\
& -(2 b(t)-\alpha)\left\|u_{t}(t)+\alpha u(t)\right\|^{2}-\alpha\left(m^{2}-\alpha^{2}\right)\|u(t)\|^{2} \\
& +2 \alpha(b(t)-\alpha)\left(u(t), u_{t}(t)+\alpha u(t)\right) \\
= & -2 \beta E_{(\alpha)}(u(t))+\frac{1}{2}\left\{a_{t}(t)-2(\alpha-\beta) a(t)\right\}\|\nabla u(t)\|^{2} \\
& -(2 b(t)-\alpha-\beta)\left\|u_{t}(t)+\alpha u(t)+\frac{\alpha(\alpha-b(t))}{2 b(t)-\alpha-\beta} u(t)\right\|^{2} \\
& -\frac{\phi(b(t), \alpha, \beta)}{2 b(t)-\alpha-\beta}\|u(t)\|^{2}
\end{aligned}
$$

where

$\phi(b(t), \alpha, \beta)=-\alpha^{2} b(t)^{2}+2\left\{m^{2}(\alpha-\beta)+\alpha^{2} \beta\right\} b(t)-m^{2}\left(\alpha^{2}-\beta^{2}\right)-\alpha^{2} \beta^{2}$.

If $2 b(t)-\alpha-\beta>0$ and $\phi(b(t), \alpha, \beta) \geq 0$ then we have

$$
\begin{aligned}
\frac{d}{d t} E_{(\alpha)} & \leq-2 \beta E_{(\alpha)}(u(t))+\frac{1}{2}\left\{a_{t}(t)-2(\alpha-\beta) a(t)\right\}\|\nabla u(t)\|^{2} \\
& \leq-2 \rho(\alpha, \beta ; I) E_{(\alpha)}(u(t)),
\end{aligned}
$$

where

$$
\rho(\alpha, \beta ; I)=\beta-\left[\sup _{t \in I}\left\{\frac{a^{\prime}(t)}{2 a(t)}\right\}-\alpha+\beta\right]+
$$

Now, we shall show that there exist $\alpha<\alpha_{0}$ and $\beta<\beta_{0}$ such that $\phi(b(t), \alpha, \beta)$ $\geq 0$ and $\rho_{0}(I)-\rho(\alpha, \beta ; I)<\varepsilon$ for any $\varepsilon>0$.

We can easily see the following inequalities $2 b(t)-\alpha-\beta>0, \phi\left(b_{0}, \alpha_{0}, \beta_{0}\right)>$ $0, \phi\left(b_{1}, \alpha_{0}, \beta_{0}\right)=0$ and $\partial_{\beta} \phi\left(b_{1}, \alpha, \beta\right)=-2\left(b_{1}-\beta\right)\left(m^{2}-\alpha^{2}\right)<0$ for any $\alpha<\alpha_{0}$ and $\beta<\beta_{0}$. Here, noting that $\phi(b, \alpha, \beta)$ is quadratic and convex with respect to $b$, hence we have $\phi(b(t), \alpha, \beta) \geq \min \left\{\phi\left(b_{0}, \alpha, \beta\right), \phi\left(b_{1}, \alpha, \beta\right)\right\}$. Therefore, by the continuity of $\phi(b, \alpha, \beta)$ with respect to $\alpha$ and $\beta$, we can take $\alpha<b$ and $\beta<b$ such that 


$$
\frac{d}{d t} E_{(\alpha)} \leq-2 \rho(\alpha, \beta ; I) E_{(\alpha)}(u(t))
$$

and

$$
\rho_{0}(I)-\rho(\alpha, \beta ; I)<\varepsilon
$$

for any given positive constant $\varepsilon$. Thus by Gronwall's inequality we have

$$
E_{(\alpha)}(u(t)) \leq E_{(\alpha)}\left(u\left(t_{0}\right)\right) \exp \left(-2\left(\rho_{0}(I)-\varepsilon\right)\left(t_{0}^{\prime}-t\right)\right)
$$

for any $t \in\left[t_{0}, t_{0}^{\prime}\right] \equiv I$ and $\varepsilon>0$. Recalling (13), we obtain

$$
E(u(t)) \leq C_{\varepsilon} E\left(u\left(t_{0}\right)\right) \exp \left(-2\left(\rho_{0}(I)-\varepsilon\right)\left(t_{0}^{\prime}-t\right)\right)
$$

and

$$
\partial_{\beta} \phi\left(b_{1}, \alpha, \beta\right)=-2\left(b_{1}-\beta\right)\left(m^{2}-\alpha^{2}\right)<0
$$

for any $\alpha<\alpha_{0}$ and $\beta<\beta_{0}$. Here, noting that $\phi(b, \alpha, \beta)$ is quadratic and convex with respect to $b$, that is, $\phi(b(t), \alpha, \beta) \geq \min \left\{\phi\left(b_{0}, \alpha, \beta\right), \phi\left(b_{1}, \alpha, \beta\right)\right\}$. Thus, on the analogy of CASE 1 , we see that there exist $\alpha<\alpha_{0}$ and $\beta<\beta_{0}$ such that $\frac{d}{d t} E_{(\alpha)} \leq$ $-2 \rho(\alpha, \beta ; I) E_{(\alpha)}(u(t))$ and $\rho_{0}(I)-\rho(\alpha, \beta ; I)<\varepsilon$ for any $\varepsilon>0$.

CASE 3. When $b_{0} b_{1} \geq m^{2}$ and $b_{0}<b_{1}$, repeating a similar proof as in CASE 2, we can also take $\alpha<\alpha_{0}$ and $\beta<\beta_{0}$ such that $\frac{d}{d t} E_{(\alpha)} \leq-2 \rho(\alpha, \beta ; I) E_{(\alpha)}(u(t))$ and $\rho_{0}(I)-\rho(\alpha, \beta ; I)<\varepsilon$ for any $\varepsilon>0$.

Therefore, by Gronwall's lemma, we obtain

$$
E_{(\alpha)}(u(t)) \leq E_{(\alpha)}\left(u\left(t_{0}\right)\right) \exp \left(-2\left(\rho_{0}(I)-\varepsilon\right)\left(t_{0}^{\prime}-t\right)\right)
$$

for any $t \in\left[t_{0}, t_{0}^{\prime}\right] \equiv I$ and $\varepsilon>0$. Recalling (13), we obtain

$$
E(u(t)) \leq C_{\varepsilon} E\left(u\left(t_{0}\right)\right) \exp \left(-2\left(\rho_{0}(I)-\varepsilon\right)\left(t_{0}^{\prime}-t\right)\right.
$$

for any positive constant $C_{\varepsilon}$. Thus, by putting $I=[0, \infty)$, the proof of Theorem 2.1 is concluded.

Remark 3.1. On the analogy of the proof above, we have also the decay estimate of higher order energy :

$$
E\left(\nabla^{j}(u(t)) \leq C_{\varepsilon} E\left(\nabla^{J} u\left(t_{0}\right)\right) \exp \left(-2\left(\rho_{0}(I)-\varepsilon\right)\left(t_{0}^{\prime}-t\right)\right)\right.
$$


for any $t \in I$ since $I \subset[0, \infty) \backslash \mathfrak{J}$.

\section{§4. Proof of Theorem 2.2}

When $a(t)$ degenerates at $t=t_{0}$, the usual hyperbolic energy $E(u(t))$ in general cannot be bounded by $E(u(0))$ in any neighborhood of $t=t_{0}$, so we shall introduce the infinity order energy.

Let $\left\{\tau_{k}\right\}$ and $\left\{\tau_{k}^{\prime}\right\}$ be sequences of non-negative real numbers satisfying $\tau_{k-1}^{\prime}<$ $\tau_{k}<t_{k}<t_{k}^{\prime}<\tau_{k}^{\prime}$ for any $k$ (if $t_{1}=0$, we put $\tau_{1}=t_{1}$ ).

Let $\delta$ be a positive real number and $\chi_{\delta}(t) \in C^{1}([0, \infty))$ be a non-negative function such that $\operatorname{supp} \chi_{\delta}(t) \subset \cup_{k \geq 1}\left[\tau_{k}, \tau_{k}^{\prime}\right], \chi_{\delta}(t)=1$ on $\mathfrak{J}_{0}$ and that $\chi_{\delta}(t)$ is monotonically increasing on $\left[\tau_{k}, t_{k}\right]$ and monotonically decreasing on $\left[t_{k}^{\prime}, \tau_{k}^{\prime}\right]$ for each $k$. We define $e_{j}(u(t))=e_{j}(u(t) ; \alpha, \delta)(j=0,1, \cdots)$ by

$$
e_{j}(u(t) ; \alpha, \delta)\left\{\begin{aligned}
= & E_{(\alpha)}(u(t)) \quad(j=0), \\
= & E_{(\alpha)}\left(\nabla^{j} u(t)\right)+j^{-1} \chi_{\delta}(t)\left\|\nabla^{j+1} u(t)\right\|^{2} \\
& +j^{3}\left(E_{(\alpha)}(u(t))+j^{-1} \chi_{\delta}(t)\left\|\nabla^{j} u(t)\right\|^{2}\right) \quad(j \geq 1) .
\end{aligned}\right.
$$

Let $\mu(t) \in C^{1}([0, \infty))$ be positive function to be chosen later. We define the infinity order energy $\mathscr{E}(u(t))=\mathscr{E}(u(t) ; \mu(t))=\mathscr{E}(u(t) ; \mu(t), \alpha, \delta)$ by

$$
\mathscr{E}(u(t) ; \mu(t), \alpha, \delta)=\sum_{j=0}^{\infty} \frac{\mu(t)^{j}}{j !^{3}} e_{j}(u(t) ; \alpha, \delta) .
$$

Differentiating $\mathscr{E}(u(t))$ with respect to $t$, we have

$$
\begin{aligned}
\frac{d}{d t} \mathscr{E}(u(t)) & =\sum_{j=0}^{\infty} \frac{\mu(t)^{j}}{j !^{3}} \frac{d}{d t} e_{j}(u(t))+\sum_{j=0}^{\infty} j \frac{\mu^{\prime}(t)}{\mu(t)} \frac{\mu(t)^{j}}{j !^{3}} e_{j}(u(t)) \\
& =\frac{d}{d t} e_{0}(u(t))+\sum_{j=1}^{\infty} \frac{\mu(t)^{j}}{j !^{3}} \frac{d}{d t} e_{j}(u(t))+\sum_{j=1}^{\infty} j \frac{\mu^{\prime}(t)}{\mu(t)} \frac{\mu(t)^{j}}{j !^{3}} e_{j}(u(t)) .
\end{aligned}
$$

Now we shall estimate the infinity order $\mathscr{E}(u(t))$. Applying Schwarz' inequality, we have

$$
\begin{aligned}
\frac{d}{d t} e_{j}(u(t))= & -2 \rho E_{(\alpha)}\left(\nabla^{j} u(t)\right)-2 j^{3} \rho E_{(\alpha)}\left(\nabla^{j-1} u(t)\right) \\
& -2 \alpha j^{-1} \chi_{\delta}(t)\left\|\nabla^{j+1} u(t)\right\|^{2}-2 \alpha j^{2} \chi_{\delta}(t)\left\|\nabla^{j} u(t)\right\|^{2} \\
& +j^{-1} \chi_{\delta}^{\prime}(t)\left\|\nabla^{j+1} u(t)\right\|^{2}+j^{2} \chi_{\delta}^{\prime}(t)\left\|\nabla^{j} u(t)\right\|^{2} \\
& +2 j^{-1} \chi_{\delta}(t)\left(\nabla^{j+1} u(t), \nabla^{j+1}\left(u_{t}(t)+\alpha u(t)\right)\right) \\
& +2 j^{2} \chi_{\delta}(t)\left(\nabla^{j} u(t), \nabla^{j}\left(u_{t}(t)+\alpha u(t)\right)\right) \\
\leq & -2 \rho e_{j}(u(t))+j^{-1}\left[\chi_{\delta}^{\prime}(t)\right]_{+}\left(\left\|\nabla^{j+1} u(t)\right\|^{2}+j^{3}\left\|\nabla^{j} u(t)\right\|^{2}\right)
\end{aligned}
$$




$$
\begin{aligned}
& +j^{-1} \chi_{\delta}(t)\left\{(j+1)\left\|\nabla^{j+1} u(t)\right\|^{2}+(j+1)^{-1}\left\|\nabla^{j+1}\left(u_{t}(t)+\alpha u(t)\right)\right\|^{2}\right\} \\
& +j^{2} \chi_{\delta}(t)\left\{j\left\|\nabla^{j} u(t)\right\|^{2}+j^{-1}\left\|\nabla^{j}\left(u_{t}(t)+\alpha u(t)\right)\right\|^{2}\right\} \\
\leq & -2\left(\rho-\frac{\left[\chi_{\delta}^{\prime}(t)\right]_{+}}{j a(t)}\right) e_{j}(u(t)) \\
& +\frac{\chi_{\delta}(t)}{j(j+1)}\left\{\left\|\nabla^{j+1}\left(u_{t}(t)+\alpha u(t)\right)\right\|^{2}+j^{2}(j+1)\left\|\nabla^{j}\left(u_{t}(t)+\alpha u(t)\right)\right\|^{2}\right\} \\
& +\chi_{\delta}(t)\left(\frac{j+1}{j}\left\|\nabla^{j+1} u(t)\right\|^{2}+j^{3}\left\|\nabla^{j} u(t)\right\|^{2}\right) \\
\leq & -2\left(\rho-\frac{\left[\chi_{\delta}^{\prime}(t)\right]_{+}+2 \chi_{\delta}(t)}{a(t)}\right) e_{j}(u(t))+\frac{4 \chi_{\delta}(t)}{j(j+1)} e_{j+1}(u(t)),
\end{aligned}
$$

where we have assumed that $\alpha \leq \beta$. Therefore, we obtain

$$
\begin{aligned}
\frac{d}{d t} \mathscr{E}(u(t) ; \mu(t)) \leq & -2\left(\rho-\eta_{\delta}(t)\right) \mathscr{E}(u(t) ; \mu(t)) \\
& +\sum_{j=1}^{\infty} \frac{\mu(t)^{j}}{j !^{3}} \frac{j}{\mu(t)}\left(\mu^{\prime}(t)+8 \chi_{\delta}(t)\right) e_{j}(u(t)),
\end{aligned}
$$

where

$$
\eta_{\delta}(t)=\frac{\left[\chi_{\delta}^{\prime}(t)\right]_{+}+2 \chi_{\delta}(t)}{a(t)}
$$

and note the inequality

$$
\sum_{j=1}^{\infty} \frac{\mu(t)^{j}}{j !^{3}} \frac{1}{j(j+1)} e_{j+1}(u(t)) \leq 2 \sum_{j=2}^{\infty} \frac{\mu(t)^{j}}{j !^{3}} \frac{j}{\mu(t)} e_{j}(u(t)) .
$$

Now, taking $\mu(t)$ as

$$
\mu(t)= \begin{cases}\mu\left(t_{k}^{\prime}\right)-8 \int_{t_{k}^{\prime}}^{t} \chi_{\delta}(s) d s & \left(t \in\left[t_{k}^{\prime}, \tau_{k}^{\prime}\right]\right), \\ \mu\left(\tau_{k}\right)-8 \int_{\tau_{k}}^{t} \chi_{\delta}(s) d s & \left(t \in\left[\tau_{k}, t_{k}\right]\right),\end{cases}
$$

we have $\mathscr{E}(u(t) ; \mu(t)) \leq\left\{\begin{array}{l}\mathscr{E}\left(u\left(t_{k}^{\prime}\right) ; \mu\left(t_{k}^{\prime}\right)\right) \exp \left(-2\left\{\rho\left(t-t_{k}^{\prime}\right)-\int_{t_{k}^{\prime}}^{t} \eta_{\delta}(s) d s\right\}\right)\left(t \in\left[t_{k}^{\prime}, \tau_{k}^{\prime}\right]\right), \\ \mathscr{E}\left(u\left(\tau_{k}\right) ; \mu\left(\tau_{k}\right)\right) \exp \left(-2\left\{\rho\left(t-\tau_{k}\right)-\int_{\tau_{k}}^{t} \eta_{\delta}(s) d s\right\}\right)\left(t \in\left[\tau_{k}, t_{k}\right]\right) .\end{array}\right.$

CASE $3\left(t \in\left[t_{k}^{\prime}, t_{k}\right]\right)$. Repeating a similar estimate as in CASE 2, we have

$$
\begin{aligned}
\frac{d}{d t} e_{0}(u(t)) \leq & -2 \beta e_{0}(u(t))+\left[\sup _{t \notin \mathfrak{J}_{0}}\left\{\frac{a^{\prime}(s)}{2 a(s)}\right\}-\alpha+\beta\right] a(t)\|\nabla u(t)\|^{2} \\
& +\frac{1}{2} a^{\prime}(t)\|\nabla u(t)\|^{2}-\sup _{t \notin \mathfrak{J}_{0}}\left\{\frac{a^{\prime}(s)}{2 a(s)}\right\} a(t)\|\nabla u(t)\|^{2}
\end{aligned}
$$




$$
\leq-2 \rho e_{0}(u(t))+\frac{\left[a^{\prime}(t)\right]_{+}}{2 \delta} e_{1}(u(t))
$$

and

$$
\begin{aligned}
\frac{d}{d t} e_{j}(u(t)) \leq & -2 \rho e_{j}(u(t))+\frac{j\left[a^{\prime}(t)\right]+}{2 \delta} e_{j}(u(t)) \\
& +\frac{\chi_{\delta}(t)}{j(j+1)}\left\{\left\|\nabla^{j+1}\left(u_{t}(t)+\alpha u(t)\right)\right\|^{2}+j^{2}(j+1)\left\|\nabla^{j}\left(u_{t}(t)+\alpha u(t)\right)\right\|^{2}\right\} \\
& +\chi_{\delta}(t)\left(\frac{j+1}{j}\left\|\nabla^{j+1} u(t)\right\|^{2}+j^{3}\left\|\nabla^{j} u(t)\right\|^{2}\right) \\
\leq & -2 \rho e_{j}(u(t))+\frac{j\left[a^{\prime}(t)\right]+}{2 \delta} e_{j}(u(t)) \\
& +\frac{4 \chi_{\delta}(t)}{j(j+1)} e_{j+1}(u(t))+2(j+1) e_{j}(u(t))
\end{aligned}
$$

Therefore, we obtain

$$
\begin{aligned}
\frac{d}{d t} \mathscr{E}(u(t) ; \mu(t)) \leq & -2 \rho \mathscr{E}(u(t) ; \mu(t)) \\
& +\sum_{j=1}^{\infty} \frac{\mu(t)^{j}}{j !^{3}} \frac{j}{\mu(t)}\left(\mu^{\prime}(t)+\left(\frac{\left[a^{\prime}(t)\right]_{+}}{2 \delta}+4\right) \mu(t)+8 \chi_{\delta}(t)\right\} e_{j}(u(t))
\end{aligned}
$$

for any $t \in\left[t_{k}, t_{k}^{\prime}\right]$. Here, taking $\mu(t)$ as

$\mu(t)=\left(u\left(t_{k}\right)-8 \int_{t_{k}}^{t} \chi_{\delta}(s) \exp \left(\int_{t_{k}}^{s} \frac{\left[a^{\prime}(\tau)\right]_{+}}{2 \delta}+4 d r\right) d s\right) \exp \left(-\int_{t_{k}}^{t} \frac{\left[a^{\prime}(s)\right]_{+}}{2 \delta}+4 d s\right)$,

we obtain

$$
\mathscr{E}\left(u(t) ; \mu(t) \leq \mathscr{E}\left(u\left(t_{k}\right) ; \mu\left(t_{k}\right)\right) e^{-2 \rho\left(t-t_{k}\right)}\right.
$$

for any $t \in\left[t_{k}, t_{k}^{\prime}\right]$.

Thus, we have the following decay estimate for the infinity order energy on $t \in\left[\tau_{k}^{\prime}, \tau_{k+1}\right]$.

$$
\begin{aligned}
\mathscr{E}(u(t) ; \mu(t)) & \leq \mathscr{E}\left(u\left(\tau_{k}^{\prime}\right) ; \mu\left(\tau_{k}^{\prime}\right)\right) e^{-2 \rho\left(t-\tau_{k}^{\prime}\right)} \\
& \leq \mathscr{E}\left(u\left(t_{k}^{\prime}\right) ; \mu\left(t_{k}^{\prime}\right)\right) \exp \left(-2 \rho\left(t-t_{k}^{\prime}\right)+\int_{t^{\prime}{ }_{k}}^{\tau^{\prime}{ }_{k}} \eta_{\delta}(s) d s\right) \\
& \leq \mathscr{E}\left(u\left(t_{k}\right) ; \mu\left(t_{k}\right)\right) \exp \left(-2 \rho\left(t-t_{k}\right)+\int_{t_{k}^{\prime}{ }_{k}}^{\tau^{\prime}} \eta_{\delta}(s) d s\right) \\
& \leq \mathscr{E}\left(u\left(\tau_{k}\right) ; \mu\left(\tau_{k}\right)\right) \exp \left(-2 \rho\left(t-\tau_{k}\right)+\int_{\left[\tau_{k}, t_{k}\right] \cup\left[t_{k}^{\prime}, \tau_{k}^{\prime}\right]} \eta_{\delta}(s) d s\right)
\end{aligned}
$$




$$
\begin{aligned}
& \leq \mathscr{E}(u(0) ; \mu(0)) \exp \left(-2 \rho t+\sum_{l=1}^{k} \int_{\left[\tau_{l}, t_{l}\right] \cup\left[t_{l}^{\prime}, \tau_{l}^{\prime}\right]} \eta_{\delta}(s) d s\right) \\
& \leq \mathscr{E}(u(0) ; \mu(0)) \exp \left(-2 \rho t+\sum_{l=1}^{k} a_{l}^{-1} \delta\left\{1+2\left(t_{l}-\tau_{l}+\tau_{l}^{\prime}-t_{l}^{\prime}\right)\right\}\right),
\end{aligned}
$$

where

$$
a_{l}^{-1}=\frac{1}{\inf _{s \in\left[\tau_{l}, t_{l}\right]}\{a(s)\}}+\frac{1}{\inf _{s \in\left[t_{l}^{\prime}, \tau_{l}^{\prime}\right]}\{a(s)\}}
$$

and we note that

$$
\begin{aligned}
\int_{\left[\tau_{k}, t_{k}\right] \cup\left[t_{k}^{\prime}, \tau_{k}^{\prime}\right]}\left[\chi_{\delta}^{\prime}(s)\right]_{+}+2 \chi_{\delta}(s) d s & \leq \chi_{\delta}\left(t_{l}\right)+2 \delta \int_{\left[\tau_{k}, t_{k}\right] \cup\left[t_{k}^{\prime}, \tau_{k}^{\prime}\right]} d s \\
& \leq \delta\left\{1+2\left(t_{l}-\tau_{l}+\tau_{l}^{\prime}-t_{l}^{\prime}\right)\right\} .
\end{aligned}
$$

Now, taking $\left\{\tau_{l}\right\}$ and $\left\{\tau_{l}^{\prime}\right\}$ satisfying that $\left(\tau_{l}^{\prime}-t_{l}^{\prime}\right)+\left(t_{l}-\tau_{l}\right) \leq 1 / 2$ for any $l$, we have

$$
\mathscr{E}(u(t) ; \mu(t)) \leq \mathscr{E}(u(0) ; \mu(0))\left(-2 \rho t+2 \delta \sum_{l=1}^{k} a_{l}^{-1}\right) .
$$

Here we remark that the decay estimate (16) holds for any $t \in\left[\tau_{k}, \tau_{k+1}\right]$.

Now, we shall estimate $\sum_{l=1}^{k} a_{l}^{-1}$ by $(8)$.

If $a^{\prime}\left(t_{l}\right)<0$, we can take $\tau_{l}$ such that $a^{\prime}(t)<0$ for any $t \in\left[\tau_{l}, t_{l}\right]$, then we have $\inf _{s \in\left[\tau_{l}, t_{l}\right]} a(s)=a\left(t_{l}\right)$. When $a^{\prime}\left(t_{l}^{\prime}\right)>0$, we can also take $\tau_{l}^{\prime}$ such that inf $\operatorname{se}_{s \in\left[t_{l}^{\prime}, \tau^{\prime}\right]} a(s)$ $=a\left(t_{l}^{\prime}\right)$.

If $a^{\prime}\left(t_{l}\right)>0$, we can take $\tau_{l}$ such that $a^{\prime}(t)>0$ for any $t \in\left[\tau_{l}, t_{l}\right]$. Then, noting that $\inf _{t \in\left[t_{l}, \tau_{l}\right]}\{a(t)\}=a\left(\tau_{l}\right)$ and $a^{\prime}(t) \leq 2 \alpha_{0} a(t)$ for any $t \in\left[\tau_{l}, t_{l}\right]$ by (7), applying the mean value theorem, there exixts $s_{l} \in\left[\tau_{l}, t_{l}\right]$ such that

$$
\begin{aligned}
\frac{1}{\inf _{t \in\left[\tau_{l}, t_{l}\right]}\{a(t)\}} & =\frac{1}{a\left(t_{l}\right)}+\frac{a\left(t_{l}\right)-a\left(\tau_{l}\right)}{a\left(t_{l}\right) a\left(\tau_{l}\right)} \\
& \leq \frac{1}{a\left(t_{l}\right)}+\frac{a^{\prime}\left(s_{l}\right)\left(t_{l}-\tau_{l}\right)}{a\left(t_{l}\right) a\left(\tau_{l}\right)} \\
& \leq \frac{1}{a\left(t_{l}\right)}+\frac{2 \alpha_{0}\left(t_{l}-\tau_{l}\right)}{a\left(t_{l}\right)} .
\end{aligned}
$$

By taking $\tau_{l}$ like $2 \alpha_{0}\left(t_{l}-\tau_{l}\right) \leq \frac{a\left(\tau_{1}\right)}{a\left(t_{l}\right)}$, we have

$$
\frac{1}{\inf _{t \in\left[\tau_{l}, t_{l}\right]}\{a(t)\}} \leq \frac{2}{a\left(t_{l}\right)} .
$$

When $a^{\prime}\left(t_{l}^{\prime}\right)<0$, we can also take $\tau_{l}^{\prime}$ satisfying 


$$
\frac{1}{\inf _{t \in\left[t_{l}^{\prime}, t_{l}^{\prime}\right]}\{a(t)\}} \leq \frac{2}{a\left(t_{l}\right)}
$$

If $a^{\prime}\left(t_{l}\right)=0$ and $a^{\prime}(t) \neq 0$ near $t<t_{l}$, or $a^{\prime}\left(t_{l}^{\prime}\right)=0$ and $a^{\prime}(t) \neq 0$ near $t>t_{l}^{\prime}$, we can also take $\tau_{l}$ and $\tau_{l}^{\prime}$ satisfying (17) and (18) respectively. that

Therefore, there exist sequences $\left\{\tau_{l}\right\}$ and $\left\{\tau_{l}^{\prime}\right\}$ of non-negative numbers such

$$
\sum_{l=1}^{k} a_{l}^{-1} \leq \sum_{l=1}^{k} 2 a\left(t_{l}\right)^{-1}+\sum_{l=1}^{k} 2 a\left(t_{l}\right)^{-1}
$$

and $\lim _{t \rightarrow \infty} \delta\left(\sum_{t_{k} \leq t} a\left(t_{k}\right)^{-1}+\sum_{t^{\prime} \leq t} a\left(t_{k}^{\prime}\right)^{-1}\right) / t=0$ for any $\delta>0$ by (8).

Thus, there exists a positive constant $C_{\delta}$ independent of $t$, we have the following decay estimate of $\mathscr{E}(u(t) ; \mu(0))$ :

$$
\mathscr{E}(u(t) ; \mu(t)) \leq C_{\delta} \mathscr{E}(u(0) ; \mu(0)) e^{-2 \rho t}
$$

for any $t \geq 0$ and $\rho<\rho_{0}$.

Next, we shall consider the positivity of $\mu(t)$. Let $t \in\left[\tau_{k}^{\prime}, \tau_{k+1}\right]$ and put $\phi_{l}(t)$ $=8 \int_{\tau_{l}}^{t} \chi_{\delta}(s) e^{\phi_{l}(s)} d s, \phi_{l}^{\prime}(t)=8 \int_{t_{l}^{\prime}}^{t_{l}} \chi_{\delta}(s) e^{\phi_{l}(s)} d s, \phi_{l}(t)=\int{ }_{t_{l}}^{t} \frac{\left[a^{\prime}(s)\right]++8 \delta}{2 \delta} d s$ and $\Psi_{l}(t)=$ $8 \int_{t_{l}}^{t} \chi_{\delta}(s) e^{\psi_{l}(s)} d s$. Recalling the definition of $\mu(t)$;

$$
\mu(\mathrm{t})= \begin{cases}\mu\left(\tau_{1}^{\prime}\right) & \left(\mathrm{t} \in\left[\tau_{1}^{\prime}, \tau_{l+1}\right]\right), \\ \mu\left(t_{l}^{\prime}\right)-\phi_{l}^{\prime}(t) & \left(t \in\left[t_{l}^{\prime}, \tau_{l}^{\prime}\right]\right), \\ e^{-\psi_{l}(t)}\left(\mu\left(t_{l}\right)-\Psi_{l}(t)\right) & \left(t \in\left[t_{l}, t_{l}^{\prime}\right]\right), \\ \mu\left(\tau_{l}\right)-\phi_{l}(t) & \left(t \in\left[\tau_{l}, t_{l}\right]\right),\end{cases}
$$

we have

$$
\begin{aligned}
\mu\left(\tau_{l+1}\right) & =\mu\left(\tau_{l}^{\prime}\right) \\
& =\mu\left(t_{l}^{\prime}\right)-\phi_{l}^{\prime} \\
& =e^{-\phi_{l}}\left\{\mu\left(t_{l}\right)-\left(\Psi_{l}+\phi_{l}^{\prime} e^{\psi_{l}}\right)\right\} \\
& =e^{-\phi_{l}}\left\{\mu\left(\tau_{l}\right)-\left(\Psi_{l}+\phi_{l}^{\prime} e^{\psi_{l}}+\phi_{l}\right)\right\}
\end{aligned}
$$

where $\phi_{l}=\phi_{l}\left(t_{l}\right), \phi_{l}^{\prime}=\phi_{l}^{\prime}\left(\tau_{l}^{\prime}\right), \phi_{l}=\phi_{l}\left(t_{l}^{\prime}\right)$ and $\Psi_{l}=\Psi\left(t_{l}^{\prime}\right)$. Therefore, we have

$$
\begin{aligned}
\mu(t) & =\mu\left(\tau_{k}^{\prime}\right) \\
& =e^{-\psi_{k}}\left\{\mu\left(\tau_{k}\right)-\left(\Psi_{k}+\phi_{k}^{\prime} e^{\psi_{k}}+\phi_{k}\right)\right\} \\
& =e^{-\phi_{k}}\left[e^{-\psi_{k-1}}\left\{\mu\left(\tau_{k-1}\right)-\left(\Psi_{k-1}+\phi_{k-1}^{\prime} e^{\psi_{k-1}}+\phi_{k-1}\right)\right\}-\left(\Psi_{k}+\phi_{k}^{\prime} e^{\psi_{k}}+\phi_{k}\right)\right]
\end{aligned}
$$




$$
\begin{aligned}
& =e^{-\left(\psi_{k}+\psi_{k-1}\right)}\left\{\mu\left(\tau_{k-1}\right)-\left(\Psi_{k} e^{\psi_{k-1}}+\Psi_{k-1}+\phi_{k}^{\prime} e^{\phi_{k}+\psi_{k-1}}+\phi_{k-1}^{\prime} e^{\psi_{k-1}}\right.\right. \\
& \left.\left.+\phi_{k} e^{\psi_{k-1}}+\phi_{k-1}\right)\right\} \\
& =e^{-\left(\psi_{k}+\phi_{k-1}+\psi_{k-2}\right)}\left\{\mu\left(\tau_{k-2}\right)-\left(\Psi_{k} e^{\phi_{k-1}+\phi_{k-2}}+\Psi_{k-1} e^{\psi_{k-2}}+\Psi_{k-2}+\phi_{k}^{\prime} e^{\psi_{k}+\psi_{k-1}+\phi_{k-2}}\right.\right. \\
& \left.\left.+\phi_{k-1}^{\prime} e^{\psi_{k-1}+\psi_{k-2}^{\prime}}+\phi_{k-2}^{\prime} e^{\phi_{k-2}}+\phi_{k} e^{\psi_{k-1}+\phi_{k-2}}+\phi_{k-1} e^{\phi_{k-1}}+\phi_{k-2}\right)\right\} \\
& =e^{-\left(\psi_{k}+\cdots+\phi_{1}\right)}\left\{\mu(0)-\left(\Psi_{k} e^{\phi_{k-1}+\cdots+\psi_{1}}+\cdots+\Psi_{2} e^{\phi_{1}}+\Psi_{1}+\phi_{k}^{\prime} e^{\psi_{k}+\cdots+\phi_{1}}\right.\right. \\
& \left.\left.+\cdots+\phi_{2}^{\prime} e^{\phi_{2}+\phi_{1}}+\phi_{1}^{\prime} e^{\phi_{1}}+\phi_{k} e^{\phi_{k-1}+\cdots+\phi_{1}}+\cdots+\phi_{2} e^{\phi_{1}}+\phi_{1}\right)\right\} \text {. }
\end{aligned}
$$

From the representation of $\mu(t)$, we can prove that $\mu(t)>0$ for any $k$ and $t$ if $\mu(0)=\mu_{0}$ is sufficiently large. Here we remark that $\mu(t)$ is monotonically increasing, hence $\mu\left(\tau_{k+1}\right) \leq \mu(t)$ for any $t \in\left[\tau_{k}, \tau_{k+1}\right]$.

Now, we choose $\left\{\tau_{l}\right\}$ and $\left\{\tau_{l}^{\prime}\right\}$ satisfying $t_{l}-\tau_{l}=\tau_{l}^{\prime}-t_{l}^{\prime} \equiv \nu_{l}$ and $\nu_{l} \leq t_{l}^{\prime}-t_{l}$. Noting that $\phi_{l} \leq 8 \delta \nu_{l}, \phi_{l}^{\prime} \leq 8 \delta \nu_{l}$ and

$$
\sum_{h=1}^{l} \phi_{h} \leq \frac{1}{2 \delta} \sum_{h=1}^{l} \int_{t_{h}}^{t_{h}^{\prime}}\left[a^{\prime}(s)\right]_{+} d s+4 \sum_{h=1}^{l}\left(t_{h}^{\prime}-t_{h}\right),
$$

we have

$$
\begin{aligned}
\sum_{l=1}^{k} \phi_{l}^{\prime} e^{\phi_{l}+\cdots+\phi_{1}} & +\sum_{l=1}^{k} \phi_{l} e^{\phi_{l-1}+\cdots+\psi_{1}} \\
& \leq \sum_{l=1}^{k}\left(\phi_{l}^{\prime}+\phi_{l}\right) \exp \left(\frac{1}{2 \delta} \sum_{h=1}^{l} \int_{t_{h}}^{t_{h}^{\prime}}\left[a^{\prime}(s)\right]_{+} d s+4 \sum_{h=1}^{l}\left(t_{h}^{\prime}-t_{h}\right)\right) \\
& \leq 16 \delta e^{4 \text { meas }\left\{\mathfrak{I}_{0}\right\}} \sum_{l=1}^{k} \nu_{l} \exp \left(\frac{1}{2 \delta} \sum_{h=1}^{l} \int_{t_{h}}^{t_{h}^{\prime}}\left[a^{\prime}(s)\right]+d s\right) \\
& \leq 16 \delta e^{4 \text { meas }\left\{\mathfrak{I}_{0}\right\}} \sum_{l=1}^{\infty} \nu_{l} \exp \left(\frac{1}{2 \delta} \sum_{h=1}^{l} \int_{t_{h}}^{t_{h}^{\prime}}\left[a^{\prime}(s)\right]_{+} d s\right) \\
& \equiv S_{\delta}<\infty
\end{aligned}
$$

and

$$
\begin{aligned}
\sum_{l=1}^{k} \Psi_{l} e^{\phi_{l-1}+\cdots+\psi_{1}} & =8 \sum_{l=1}^{k} \int_{t_{h}}^{t^{\prime}} \chi_{\delta}(s) e^{\psi_{l}(s)} d s e^{\psi_{l-1}+\cdots+\phi_{1}} \\
& \leq 8 \sum_{l=1}^{k}\left(t_{l}^{\prime}-t_{l}\right) e^{\psi_{l}+\cdots+\phi_{1}} \\
& \leq 8 e^{4 \operatorname{meas}\left\{\mathfrak{I}_{0}\right\}} \sum_{l=1}^{k}\left(t_{l}^{\prime}-t_{l}\right) \exp \left(\frac{1}{2 \delta} \sum_{h=1}^{l} \int_{t_{h}}^{t^{\prime} h}\left[a^{\prime}(s)\right]_{+} d s\right) \\
& \equiv S_{\delta}^{\prime}<\infty
\end{aligned}
$$

by (9), where meas $\left\{\mathfrak{I}_{0}\right\}$ is the measure of $\mathfrak{I}_{0}$. Therefore, we obtain 


$$
\mu(t) \geq e^{-\Sigma_{k} \psi_{k}}\left\{\mu_{0}-\left(S_{\delta}+S_{\delta}^{\prime}\right)\right\}>0
$$

for any $t \in\left[\tau_{k}, \tau_{k+1}\right](k=1,2, \cdots)$ since $\mu_{0}>S_{\delta}+S_{\delta}^{\prime}$.

Here we remark that $\mu(t)$ is positive for any $t \geq 0$, but $\mu(t)$ is not uniformly positive, because $\sum_{l=1}^{k} \phi_{l} \rightarrow \infty$ as $k \rightarrow \infty$ in general. On the other hand, if (10) is satisfied, then $\sum_{l=1}^{\infty} \psi_{l}<\infty$, hence, $\mu(t)$ is uniformly positive, that is, there exists a positive constant $\mu_{\infty}$ such that $\inf _{t} \mu(t)=\lim _{t \rightarrow \infty} \mu(t)=\mu_{\infty}$.

Now, we shall conclude the proof of Theorem 2.2 from the decay estimate (19).

We introduce the following lemma.

Lemma 4.1. Let $0<s \leq 3 / 2$ and $\mu>0$.

(i) If $u_{0}, u_{1} \in G^{s}$, then the infinity order energy $\mathscr{E}\left(u(0) ; \mu_{0} ; \alpha, \delta\right)$ is finite for any $\mu_{0}>0, \alpha<m$ and $\delta>0$.

(ii) There exists a positive constant $C_{\alpha, \delta}$ such that $\sum_{j=0}^{\infty} \frac{\mu^{j}}{j !^{3}} E\left(\nabla^{j} u(t)\right)=$ $\mathscr{E}_{0}(u(t) ; \mu, 0,0) \leq C_{\alpha, \delta} \mathscr{E}(u(t) ; \mu(t), \alpha, \delta)$.

Proof. (i) Noting the inequality (12), we have

$$
\begin{aligned}
\mathscr{E}\left(u(0) ; \mu_{0}, \alpha, \delta\right) \leq & C_{\alpha} \sum_{j=0}^{\infty} \frac{\mu_{0}^{j}}{j !^{3}} E\left(\nabla^{j} u(0)\right) \\
& +\frac{1}{2} \delta \sum_{j=1}^{\infty} \frac{\mu_{0}^{j}}{j !^{3}}\left(j^{-1}\left\|\nabla^{j} u(0)\right\|^{2}+j^{2}\left\|\nabla^{j-1} u(0)\right\|^{2}\right) \\
\leq & C_{1} C_{\alpha} \max \left\{1, m^{2}-\alpha^{2}, a(0), \frac{\delta}{2}\right\} \sum_{j=0}^{\infty}\left(\frac{\mu_{0}}{\mu^{2}}\right)^{j} j !^{-3+2 s} \\
& <\infty
\end{aligned}
$$

where $\mu$ is a positive constant and we used the inequality $\frac{\kappa^{\jmath}}{j !} \leq e^{\kappa}$ for any positive integer $j$ and any positive real number $\kappa$.

(ii) is trivial from the inequality (12).

Therefore, we obtain

$$
E(u(t)) \leq \sum_{j=0}^{\infty} \frac{\mu(t)^{j}}{j !^{3}} E\left(\nabla^{j} u(t)\right) \leq C_{\rho, \delta} \mathscr{E}(u(0) ; \mu(0), \alpha) e^{-2 \rho t} .
$$

In particular, if $\mu(t)$ is uniformly positive, then we have the following decay estimate for the infinity order energy :

$$
\sum_{j=0}^{\infty} \frac{\mu_{\infty}^{j}}{j !^{3}} E\left(\nabla^{j} u(t)\right) \leq C_{\rho, \delta} \mathscr{E}(u(0) ; \mu(0), \alpha) e^{-2 \rho t}
$$

for any $\rho<\rho_{0}$. Thus, the proof of Theorem 2.2 is concluded. 
Theorem 2.3 does not follow from the decay estimate (20) without the assumption (10). However, by considering the infinity order energy $\mathscr{E}\left(\nabla^{k} u(t)\right.$; $\mu(t))$, we have a decay estimate of the higher order energy $E\left(\nabla^{k} u(t)\right)$ by analogy with the proof of Theorem 2.2 .

\section{§5. Examples}

Finally, we shall introduce some examples which can be applied our theorems.

Example 1. Let $p>1$ and $q>0$, and define $a(t)$ by

$$
a(t)=\sin (q t)+p, \quad b(t) \equiv 1 \text { and } m=1 .
$$

If $\sup _{t}\left\{\frac{a^{\prime}(t)}{2 a(t)}\right\}=\sup _{t}\left\{\frac{q \cos (q t)}{2(\sin (q t)+p)}\right\}=\frac{q}{2(p-1)}<\beta_{0}=1$, then the total energy of the solution satisfies the following decay estimate :

$$
E(u(t)) \leq C_{\rho} E(u(0)) e^{-2 \rho t}
$$

for any $t \geq 0$ and $\rho<\rho_{0}=1-q / 2(p-1)$.

Example 2. Let $p \geq 1$ and define

$$
a(t)=t^{p}, \quad b(t)=1 \text { and } m=1 .
$$

Noting that $\sup _{t>p / 2}\left\{\frac{a^{\prime}(t)}{2 a(t)}\right\}=\sup _{t>p / 2}\left\{\frac{p}{2 t}\right\}<\beta_{0}=1$, we see that $\mathfrak{I}=[0, p / 2]$. Now we let $\mathfrak{I}_{0}=[0, p \tau / 2]$, then $\mathfrak{I}_{0}$ satisfies from (7) to (10) for any $\tau>1$. If $u_{0}, u_{1} \in$ $G^{s}(s<3 / 2)$, then there exists a positive constant $C_{\rho, u_{0}, u_{1}}$ such that the total energy of the solution satisfies the following decay estimate :

$$
E(u(t)) \leq C_{\rho, u_{0}, u_{1}} E(u(0)) e^{-2 \rho t}
$$

for any $t \geq 0$ and $\rho<\rho_{0}=1-\tau^{-1}$. Moreover, we can take $\tau>1$ arbitrary and the decay estimate above holds for any $\rho<1$.

Example 3. Let $p$ and $q$ be positive constants satisfying $a+q / p<p$. Define $a(t) \in C^{1}([0, \infty))$ by 


$$
a(t)= \begin{cases}\frac{\cos \left(\left(2 \pi e^{j}(t-j)\right)+1\right.}{[p] \sqrt{1+t}} & \left(t \in\left[j, j+e^{-j}\right] \quad(j=1,2, \cdots)\right), \\ \frac{1}{[p] \sqrt{1+t}} & \left(t \notin\left[j, j+e^{-j}\right] \quad(j=1,2, \cdots)\right),\end{cases}
$$

$b(t)=2$ and $m=3$. Put $\left\{t_{j}\right\}=\left\{j^{q}\right\}$ and $\left\{t_{j}^{\prime}\right\}=\left\{j^{q}+e^{-j}\right\}$. Then, the assumptions in Theorem $2.2(7)-(9)$ are fulfilled. Indeed, $\mathfrak{J} \subset \cup\left(t_{j}, t_{j}^{\prime}\right) \equiv \mathfrak{I}_{0}$,

$$
\begin{gathered}
\operatorname{meas}\left\{\mathfrak{J}_{0}\right\}=\sum_{j=1}^{\infty} e^{-j}=\frac{1}{e-1}<\infty, \\
\frac{\sum_{\left\{k ; t_{k} \leq t\right\}} a\left(t_{k}\right)^{-1}+\sum_{\left\{k ; t_{k}^{\prime} \leq t\right\}} a\left(t_{k}^{\prime}\right)^{-1}}{t} \leq \frac{2 \sum_{j=1}^{k}\left(j^{\frac{q}{p}}+2\right)}{k^{p}}=\frac{\mathcal{O}\left(1+k^{\frac{q}{p}+1}\right)}{k^{p}} \rightarrow 0 \\
\quad(k \rightarrow \infty)
\end{gathered}
$$

for any $t \in\left[k^{q},(k+1)^{q}\right)$, and

$$
\begin{aligned}
\sum_{j=1}^{\infty}\left(t_{j}^{\prime}-t_{j}\right) \exp \left(M \sum_{l=1}^{j} \int_{t_{l}}^{t_{l}^{\prime}}\left[a^{\prime}(s)\right]_{+} d s\right) & =\sum_{j=1}^{\infty} \exp \left(-j+M \sum_{l=1}^{j} \int_{t_{l}}^{t_{l}^{\prime}}\left[a^{\prime}(s)\right]_{+} d s\right) \\
& \leq\left\{\begin{array}{ll}
\sum_{j=1}^{\infty} e^{-j+M j^{1-\frac{q}{p}}} & \left(\frac{q}{p} \neq 1\right) \\
\sum_{j=1}^{\infty} j^{M} e^{-j}<\infty & \left(\frac{q}{p}=1\right)
\end{array}\right\}<\infty
\end{aligned}
$$

for any $M>0$. By noting that $\sup _{t \in[0, \infty) \backslash \mathfrak{I}_{0}} \frac{a^{\prime}(t)}{2 a(t)} \leq 0$, there exists a positive constant $C_{\rho, u_{0}, u_{1}}$ such that the total energy of the solution to (1)-(2) has the decay estimate of exponential order $E(u(t)) \leq C_{\rho, u_{0}, u_{1}} e^{-2 \rho t}$ for any $t \geq 0$ and $\rho<2$ since $u_{0}, u_{1} \in G^{s}$ with $s<3 / 2$.

\section{References}

[1] Colombini, F., Jannelli, E. and Spagnolo, S., Well-Posed in the Gevrey classes of the Cauchy problem for a non-strictly hyperbolic equation with coefficients depending on time, Ann. Scuola Norm. Sup. Pisa Cl. Sci. (4), 10(1983), 291-312.

[2] Matsumura, A., Energy decay of solutions of dissipative wave equations, Proc. Japan Acad. Ser. A Math. Sci., 53(1977), 232-236.

[ 3 ] Mochizuki, K., Scattering theory for wave equations (Japanese), Kinokuniya, 1984.

[4] Mochizuki, K. and Nakazawa, H., Energy decay and asymptotic behavior of solutions to the wave equations with linear dissipation, Publ. RIMS, 32 (1996), 401-414.

[ 5 ] Rauch, J. and Taylor, M., Decreasing states of perturbed wave equations, J. Math. Anal. Appl., 54(1976), 279-285.

[6] Reissig, M. and Yagdjian, K., One application of Floquet's theory to $L_{p}-L_{q}$ decay estimates, Math. Methods Appl. Sci., to appear.

[7] Zuazua, E., Stability and decay for a class of nonlinear hyperbolic problems, Asympto. Anal., 1 (1988), 161-185. 